\title{
COMPLETELY MONOTONIC FUNCTIONS ON $n$-DIMENSIONAL LATTICES*
}

\author{
JOHAN HAVNEN
}

\section{Introduction.}

The Cartesian product $L$ of $n$ linearly ordered sets $L_{i}$ is a partially ordered set with respect to the coordinatewise ordering. This partial ordering imposes a lattice structure on $L$ and $(L ; \wedge)$ is an idempotent semigroup. We investigate the nature of the completely monotonic (CM) functions on this semigroup and are able to give a sufficient condition for $f: L \rightarrow \mathrm{R}$ to be a CM-function. To do this we restrict our attention to a certain convex cone $C(L)$ of real valued functions, which satisfy two conditions $(3.2$ (i) and (ii)). We are able to identify the extreme points of a base of this cone as exponentials [2] and thus show that $C(L)$ is an extremal subcone of the cone of CM-functions, $C_{\infty}(L)$, on $(L ; \wedge)$. This enables us in section 4 to show that a sufficient condition for $f: L \rightarrow \mathrm{R}$ to be a CM-function on $(L ; \wedge)$ is that $\Delta_{k} f \geqq 0,0 \leqq k \leqq n$ (for definition see section 2). We also decompose every $f \in X_{\infty}(L)$ into a certain type of convex sums.

\section{Preliminaries.}

If $S$ denotes a commutative semigroup with identity $e$ and if $f: S \rightarrow R$, then the difference operators $\Delta_{n}$, for $n$ nonnegative integer, are defined inductively by $\Delta_{0} f(x)=f(x)$ and

$$
\Delta_{n} f\left(x_{0} ; x_{1}, \ldots, x_{n}\right)=\Delta_{n-1} f\left(x_{0} ; x_{1}, \ldots, x_{n-1}\right)-\Delta_{n-1} f\left(x_{0} x_{n} ; x_{1}, \ldots, x_{n-1}\right) \text {. }
$$

The function $f$ is said to be completely monotonic if $\Delta_{n} f\left(x_{0} ; x_{1}, \ldots, x_{n}\right) \geqq 0$ for all choices of $x_{0}, x_{1}, \ldots, x_{n} \in S$ and all nonnegative integers $n$. Let $C_{\infty}(S)$ denote the family of all completely monotonic (CM) functions on $S$ and

$$
X_{\infty}(S)=\left\{f \in C_{\infty}(S) \mid f(e)=1\right\} \text {. }
$$

Received December 19, 1972.

*The result of this paper is contained in the authors Ph.D.-thesis at the Pennsylvania State University written under the direction of Professor P. H. Maserick. 
Then $C_{\infty}(S)$ is a convex cone with base [7] $X_{\infty}(S)$ in the linear space $\mathrm{R}^{S}$ of all real valued functions on $S$. If $R^{S}$ is equipped with the topology of pointwise convergence, then the span $E_{\infty}(S)=C_{\infty}(S)-C_{\infty}(S)$ of $C_{\infty}(S)$ becomes a locally convex linear topological space and $X_{\infty}(S)$ is compact. It is known from [4] that $X_{\infty}(S)$ is an $r$-simplex, that is every $f \in X_{\infty}(S)$ admits a unique representing measure which is supported by the extreme points $\left(\operatorname{ext} X_{\infty}(S)\right)$ of $X_{\infty}(S)$, and $\operatorname{ext} X_{\infty}(S)$ is closed.

\section{An extremal subcone of $C_{\infty}(L)$.}

In the following we consider the Cartesian product $L$ of $n$ linearly ordered sets $L_{i}$, each with a smallest element, $o_{i}$, and a largest element, $e_{i}$. We will leave out the indices when no misunderstanding may arise. Then $L=\prod_{i=1}^{n} L_{i}$ becomes a lattice if $x \vee y=\left(x_{1} \vee y_{1}, \ldots, x_{n} \vee y_{n}\right)$ where $x_{i} \vee y_{i}=x_{i}$ if $x_{i} \geqq y_{i}$ and $x_{i} \vee y_{i}=y_{i}$ if $x_{i}<y_{i}$ and $x \wedge y$ is defined similarly. Moreover $(L ; \wedge)$ is an idempotent semigroup with identity $e=(e, \ldots e)$.

Lemma 3.1. Let $n \geqq 2$. Given $x^{1}, \ldots, x^{n} \in L$ such that

(i) $x^{k}=\left(x_{1}, \ldots, x_{k-1}, x_{k}^{k}, x_{k+1}, \ldots, x_{n}\right) \quad$ where $x_{k}^{k} \leqq x_{k}, 1 \leqq k \leqq n$.

Then

$$
\bigvee_{i=1}^{n} x^{i}=\left(x_{1}, \ldots, x_{n}\right), \quad \bigwedge_{i=1}^{n} x^{i}=\left(x_{1}{ }^{1}, \ldots, x_{n}{ }^{n}\right)
$$

and if $y \leqq \bigvee_{i=1}^{n} x^{i}$ and $y \nless x^{k}$ for every $k$ then $\wedge_{i=1}^{n} x^{i} \leqq y$.

Definition 3.2. If $n \geqq 2$ let $C(L)$ denote the set of real valued functions on $L$ such that

(i) $\Delta_{n} f\left(\bigvee_{i=1}^{n} x^{i} ; x^{1}, \ldots, x^{n}\right) \geqq 0 \quad$ whenever the collection $x^{1}, \ldots, x^{n}$ satisfies 3.1.(i).

(ii) $f(x)=0 \quad$ whenever for some $k, x_{k}=o$,

and let $X(L)$ denote the set $\{f \in C(L) \mid f(e)=1\}$.

If $n=1$ then $C(L)$ denotes the set of increasing functions such that $f(o)=0$.

Proposition 3.3. The set $C(L)$ is a closed convex cone with compact base $X(L)$ in the space $\mathrm{R}^{L}$ equipped with the topology of pointwise convergence.

Proof. We will show that $X(L)$ is a compact base. The function $\hat{e}$ as defined by $\hat{e}(f)=f(e)$ is a continuous linear functional,

$$
H=\{f: L \rightarrow \mathrm{R} \mid \hat{e}(f)=1\}
$$


is a hyperplane missing the origin and $X(L)=H \cap C(L)$. Let $f \in C(L)$, $f \neq 0$, and let $x \in L$ such that $f(x) \neq 0$.

By 3.2 (i) and (ii)

$$
\Delta_{n} f\left(x ;\left(o, x_{2}, \ldots, x_{n}\right), \ldots,\left(x_{1}, \ldots, x_{n-1} o\right)\right)=f(x)>0 .
$$

Moreover $\Delta_{1} f(y ; o) \geqq 0$ for every $y \in L$. To show that $f(e) \geqq f(x)$ we observe that by 3.2 (i) and (ii)

$$
\begin{array}{r}
\Delta_{n} f\left(e ;(o, e, \ldots, e), \ldots,(e, \ldots e, o, e),\left(e, \ldots, e, x_{n}\right)\right) \\
=f(e)-f\left(e, \ldots, e, x_{n}\right) \geqq 0 .
\end{array}
$$

Similarly we show that

$$
f\left(e, \ldots, e, x_{n-k}, \ldots, x_{n}\right) \geqq f\left(e, \ldots, e, x_{n-k-1}, x_{n-k}, \ldots, x_{n}\right)
$$

and hence it follows that $f(e) \geqq f(x)$. More generally it follows that $\Delta_{1} f(e ; y) \geqq 0$ for all $y \in L$. Hence $g=f / f(e) \in X(L)$ and so $X(L)$ is a base. Since $0 \leqq f(y) \leqq 1$ whenever $f \in X(L)$ and $X(L)=H \cap C(L)$ it follows from Tychonoffs theorem that $X(L)$ is a closed subset of a compact, and hence compact.

Lemma 3.4. Let $n \geqq 2$. Given a collection $x^{1}, \ldots, x^{n} \in L$ which satisfies 3.1 (i) and let $x^{n+1} \leqq \mathrm{~V}_{i=1}^{n} x^{i}$. Then

$$
\Delta_{n+1} f\left(\bigvee_{i=1}^{n} x^{i} ; x^{1}, \ldots, x^{n}, x^{n+1}\right) \geqq 0
$$

whenever $f \in C(L)$.

Proof. Consider first the case that for some integer $x^{n+1}<x^{i}$. Direct calculations then show that

$$
\Delta_{n+1} f\left(\bigvee_{i=1}^{n} x^{i} ; \ldots, x^{n}, x^{n+1}\right)=\Delta_{n} f\left(\bigvee_{i=1}^{n} x^{i} ; x^{1}, \ldots, x^{n}\right) \geqq 0 .
$$

Suppose therefore that $x^{n+1} \nless x^{k}$ for each $k$. Then by Lemma 3.1 $\wedge_{i=1}^{n} x^{i} \leqq x^{n+1}$. Assume $x^{k} \leqq x^{n+1}$ for some $k$, say $n=k$. Then since $x^{n} \wedge x^{n+1}=x^{n}$,

$$
\begin{aligned}
\Delta_{n+1} f\left(\bigvee_{i=1}^{n}\right. & \left.x^{i} ; x^{1}, \ldots, x^{n}, x^{n+1}\right) \\
= & \Delta_{n} f\left(\bigvee_{i=1}^{n} x^{i} ; x^{1}, \ldots, x^{n}\right)-\Delta_{n} f\left(x^{n+1} ; x^{1}, \ldots, x^{n}\right) \\
= & \Delta_{n-1} f\left(\bigvee_{i=1}^{n} x^{i} ; x^{1}, \ldots, x^{n-1}\right)-\Delta_{n-1} f\left(x^{n} ; x^{1}, \ldots, x^{n-1}\right) \\
& -\Delta_{n-1} f\left(x^{n+1} ; x^{1}, \ldots, x^{n-1}\right)+\Delta_{n-1} f\left(x^{n} ; x^{1}, \ldots, x^{n-1}\right) \\
= & \Delta_{n} f\left(\bigvee_{i=1}^{n} x^{i} ; x^{1}, \ldots, x^{n-1}, x^{n+1}\right) .
\end{aligned}
$$


Hence

$$
\begin{aligned}
\Delta_{n+1} f\left(\bigvee_{i=1}^{n} x^{i} ; x^{1}, \ldots, x^{n}, x^{n+1}\right) \\
\quad=\Delta_{n} f\left(\bigvee_{i=1}^{n} x^{i} ; x^{1}, \ldots, x^{k-1}, x^{k+1}, \ldots, x^{n+1}\right)
\end{aligned}
$$

whenever for some $k, x^{k} \leqq x^{n+1}$. The collection $x^{1}, \ldots, x^{k-1}, x^{n+1}, x^{k+1}, \ldots$, $x^{n}$ satisfies 3.1 (i) and hence by 3.2 (i) and (1) it follows that

$$
\Delta_{n+1} f\left(\bigvee_{i=1}^{n} x^{i} ; x^{1}, \ldots, x^{n}, x^{n+1}\right) \geqq 0
$$

whenever $x^{k} \leqq x^{n+1} \leqq \mathrm{~V}_{i=1}^{n} x^{i}$ for some $k$. We define

$$
\begin{aligned}
& z^{0}=\bigvee_{i=1}^{n} x^{i}, \quad z^{1}=\left(x_{1}, \ldots, x_{n-1}, x_{n}{ }^{n+1}\right), \ldots, \\
& z^{k}=\left(x_{1}, \ldots, x_{n-k}, x_{n-k+1}^{n+1}, \ldots, x_{n}{ }^{n+1}\right), \ldots, \quad z^{n}=x^{n+1} .
\end{aligned}
$$

Then $x^{n} \leqq z^{1} \leqq \mathrm{~V}_{i=1}^{n} x^{i}$ and hence by (2)

that is

$$
\Delta_{n+1} f\left(\bigvee_{i=1}^{n} x^{i} ; x^{1}, \ldots, x^{n}, z^{1}\right) \geqq 0
$$

$$
\begin{aligned}
\Delta_{n} f\left(\bigvee_{i=1}^{n} x^{i} ; x^{1}, \ldots, x^{n}\right) & \geqq \Delta_{n} f\left(z^{1} ; x^{1}, \ldots, x^{n}\right) \\
& =\Delta_{n} f\left(z^{1} ; z^{1} \wedge z^{1}, \ldots, x^{n} \wedge z^{1}\right)
\end{aligned}
$$

The collection $x^{1} \wedge z^{1}, \ldots, x^{n} \wedge z^{1}$ satisfies 3.1 (i) and

$$
x^{n-1} \wedge z^{1} \leqq z^{2} \leqq \bigvee_{i=1}^{n}\left(x^{i} \wedge z^{1}\right)=z^{1}
$$

and hence by (2)

that is

$$
\Delta_{n+1} f\left(z^{1} ; x^{1} \wedge z^{1}, \ldots, x^{n} \wedge z^{1}, z^{2}\right) \geqq 0
$$

$$
\Delta_{n} f\left(z^{1} ; x^{1} \wedge z^{1}, \ldots, x^{n} \wedge z^{1}\right) \geqq \Delta_{n} f\left(z^{2} ; x^{1} \wedge z^{2}, \ldots, x^{n} \wedge z^{2}\right) .
$$

Similarly we show that

$$
\begin{aligned}
& \Delta_{n} f\left(z^{k} ; x^{1} \wedge z^{k}, \ldots, x^{n} \wedge z^{k}\right) \\
& \quad \geqq \Delta_{n} f\left(z^{k+1} ; x^{1} \wedge z^{k+1}, \ldots, x^{n} \wedge z^{k+1}\right), \quad 0 \leqq k \leqq n-1 .
\end{aligned}
$$

These inequalities (3) give us

$$
\begin{aligned}
& \Delta_{n} f\left(\bigvee_{i=1}^{n} x^{i} ; x^{1}, \ldots, x^{n}\right) \geqq \Delta_{n} f\left(z^{1} ; x^{1} \wedge z^{1}, \ldots, x^{n} \wedge z^{1}\right) \\
& \quad \geqq \ldots \geqq \Delta_{n} f\left(z^{n} ; x^{1} \wedge z^{n}, \ldots, x^{n} \wedge z^{n}\right) \\
& \quad=\Delta_{n} f\left(x^{n+1} ; x^{1}, \ldots, x^{n}\right)
\end{aligned}
$$

that is

$$
\Delta_{n+1} f\left(\bigvee_{i=1}^{n} x^{i} ; x^{1}, \ldots, x^{n}, x^{n+1}\right) \geqq 0
$$


Lemma 3.5. If $f \in C(L)$ and $g$ is defined by $g(y)=f(x \wedge y), x$ a fixed element of $L$, then $f-g \in C(L)$, that is, $f \geqq g$ in the ordering induced by the cone $C(L)$.

Proof. Since the case $n=1$ is trivially established let $n \geqq 2$. Given a collection $x^{1}, \ldots, x^{n} \in L$ which satisfies 3.1 (i) and $x^{n+1}=\left(\mathrm{V}_{i=1}^{n} x^{i}\right) \wedge x$, where $x$ is a fixed element of $L$. Since $\mathrm{V}_{i=1}^{n}\left(x^{i} \wedge x\right)=x^{n+1}$ and $x^{i} \wedge x=$ $x^{i} \wedge x^{n+1}$ it follows that

$$
\begin{aligned}
& \Delta_{n}(f-g)\left(\bigvee_{i=1}^{n} x^{i} ; x^{1}, \ldots, x^{n}\right) \\
& \quad=\Delta_{n} f\left(\bigvee_{i=1}^{n} x^{i} ; x^{1}, \ldots, x^{n}\right)-\Delta_{n} f\left(x^{n+1} ; x^{1} \wedge x^{n+1}, \ldots, x^{n} \wedge x^{n+1}\right) \\
& \quad=\Delta_{n+1} f\left(\bigvee_{i=1}^{n} x^{i} ; x^{1}, \ldots, x^{n}, x^{n+1}\right)
\end{aligned}
$$

Hence by Lemma 3.4, $f-g \in C(L)$. To see that $g \in C(L)$ observe that if the collection $\left\{x^{i}\right\}_{i=1}^{n}$ satisfies 3.1 (i) then so does $\left\{x^{i} \wedge x\right\}_{i=1}^{n}$. Hence $g$ satisfies 3.2 (i) since

$$
\begin{aligned}
\Delta_{n} f\left(\bigvee_{i=1}^{n}\left(x^{i} \wedge x\right)\right. & \left.; x^{1} \wedge x, \ldots, x^{n} \wedge x\right) \\
& =\Delta_{n} g\left(\bigvee_{i=1}^{n} x^{i} ; x^{1}, \ldots, x^{n}\right) .
\end{aligned}
$$

It therefore follows that $f \geqq g$.

Recall that $C_{\infty}(L)$ denotes the cone of CM-functions on $(L ; \Lambda)$ and $X_{\infty}(L)$ is a compact base of $C_{\infty}(L)$ which is an $r$-simplex.

Theorem 3.6. $C(L)$ is an extremal subcone of $C_{\infty}(L)$ and $X(L)$ is a closed face of $X_{\infty}(L)$, hence an r-simplex.

Proof. Let $f \in \operatorname{ext} X(L)$. By Lemma 3.5 then $f \geqq g$ where $g(y)=$ $f(x \wedge y)$ and $x$ is a fixed element of $L$. Direct calculations show that $g \in C(L)$. Since $f$ is an extreme point, $g$ therefore, must be a multiple of $f$, that is there exists an $\alpha>0$ such that $g=\alpha f$. Evaluating $g$ at $e$ gives $f(x)=\alpha$.

Hence $f(x \wedge y)=f(x) f(y)$ which implies that $f$ is an exponential. Thus from [2] it follows that $\operatorname{ext} X(L) \subset \operatorname{ext} X_{\infty}(L)$ which implies that

$$
\operatorname{co}(\operatorname{ext} X(L)) \subset \operatorname{co}\left(\operatorname{ext} X_{\infty}(L)\right) .
$$

By the Krein-Milman theorem [3]

$$
X(L)=\overline{\operatorname{co}}(\operatorname{ext} X(L)), \quad X_{\infty}(L)=\overline{\operatorname{co}}\left(\operatorname{ext} X_{\infty}(L)\right)
$$

which means that $X(L) \subset X_{\infty}(L)$ and $C(L) \subset C_{\infty}(L)$. Routine checking shows that $X(L)$ is a closed face of $X_{\infty}(L)$. Since $X(L)$ is compact and 
convex it follows from the Krein-Milman theorem [7] in the integral representation form that there exists a representing measure $\mu_{f}$ supported by ext $X(L)$. But $X(L)$ is a closed face of the $r$-simplex $X_{\infty}(L)$ so that $\mu_{f}$ is unique. Hence $X(L)$ is an $r$-simplex.

REMARK. If $L_{i}=\left[a_{i}, b_{i}\right]$ is an interval of the extended real numbers then the collection of cumulative distribution functions [8] is a subset of $X(L)$, that is, any cumulative distribution functions is a completely monotonic function with respect to that semigroup operation. An example, due to Munroe [6] shows that the boundary condition 3.2 (ii) is essential.

4. A decomposition of $\boldsymbol{C}_{\infty}(L)$.

Proposition 4.1. Let

$$
C_{n}(L)=\left\{f: L \rightarrow \mathrm{R} \mid \Delta_{k} f\left(x^{0} ; x^{1}, \ldots, x^{k}\right) \geqq 0,0 \leqq k \leqq n, x^{i} \in L\right\} .
$$

Then $C_{n}(L)$ is a closed convex cone with base

$$
X_{n}(L)=\left\{f \in C_{n}(L) \mid f(e)=1\right\} .
$$

We omit the proof which is similar to that of 3.3 .

Definition 4.2. Fix an index $j \in\{0,1, \ldots, n\}$ and $x \in L$. For each $i \leqq\left(\begin{array}{c}n \\ j\end{array}\right)$ let $x_{i, j}$ be that member of $L$ whose coordinate values agree with the coordinate values of $x$ in $j$ given coordinates and are zero elsewhere. The selection of the $j$ coordinates where agreement occurs, is the same for all $x$, dependent on $i$ and distinct for distinct $i$. Thus $i$ ranges over the set $1,2, \ldots,\left(\begin{array}{c}n \\ j\end{array}\right)$. Let

$$
G_{1,0}=\left\{f \in X_{n}(L) \mid f(o)=0\right\} .
$$

For each positive integer $p \leqq\left(\begin{array}{c}n \\ j\end{array}\right),(j=0,1, \ldots, n)$, let

and

$$
H_{p, j}=\bigcap_{i=1}^{p}\left\{f \in X_{n}(L) \mid f\left(x_{i, j}\right)=0, \forall x \in L\right\}
$$

$$
G_{l, k}=\left(\bigcap_{j=1}^{k-1} H_{\left(\begin{array}{c}
n \\
j
\end{array}\right), j}\right) \cap H_{l, k}, \quad 1 \leqq k \leqq n, 1 \leqq l \leqq\left(\begin{array}{c}
n \\
k
\end{array}\right) .
$$

Let

$$
\begin{aligned}
G_{0, k} & =G_{\left(\begin{array}{c}
n \\
k-1
\end{array}\right), k-1}, \quad 1 \leqq k \leqq n, \\
F_{l, k} & =\left\{f \in X_{n}(L) \mid f(x)=f\left(x_{l, k}\right)\right\} \cap G_{l-1, k}
\end{aligned}
$$

and $F_{1,0}=\{$ identically 1 -function $\}$. 
Some properties of the sets $G_{l, k}$ and $F_{l, k}$ are as follows:

(a) $G_{l, k}, F_{l, k} \subset G_{l-1, k}$.

(b) If $x_{l, k}$ has more than $n-k$ zero coordinate values and $f \in F_{l, k}$ then $f(x)=0$.

(c) $F_{l, k} \cap G_{l, k}=\emptyset$.

(d) $F_{l, k} \cap F_{l^{\prime}, k^{\prime}}=\emptyset$ if $(l, k) \neq\left(l^{\prime}, k^{\prime}\right)$.

(e) $G_{1, n}=\varnothing$.

Lemma 4.3. Let $\left\{G_{l, k}\right\}$ and $\left\{F_{l, k}\right\}$ be collections of subsets of $X_{n}(L)$ according to 4.2. Then $F_{l, k}$ and $G_{l, k}$ are closed, convex and extremal with respect to $G_{l-1, k}$.

Moreover the sets $G_{l, k}$ and $F_{l, k}$ are complemented in $L_{l-1, k}$ when $0 \leqq k \leqq n$ and $1 \leqq l \leqq\left(\begin{array}{c}n \\ k\end{array}\right)$.

Proof. The sets $F_{l, k}$ and $G_{l, k}$ are trivially closed and convex. That $G_{l, k}$ and $F_{l, k}$ are extremal in $G_{l-1, k}$ follows from the nonnegativity of $\Delta_{0} f$ and $\Delta_{1} f$ respectively. Direct calculations show that if

$$
f \in G_{l-1, k}-G_{l, k} \cup F_{l, k}
$$

then $0<f\left(e_{l, k}\right)<1$ because $\Delta_{0} f \geqq 0, \Delta_{1} f \geqq 0$ and $\Delta_{2} f \geqq 0$. Hence

$$
f(x)=\left(1-f\left(e_{l, k}\right)\right) \frac{f(x)-f\left(x_{l, k}\right)}{1-f\left(e_{l, k}\right)}+f\left(e_{l, k}\right) \frac{f\left(x_{l, k}\right)}{f\left(e_{l, k}\right)}
$$

which is the desired convex combination.

REMark. We only used the properties $\Delta_{0} f \geqq 0, \Delta_{1} f \geqq 0$ and $\Delta_{2} f \geqq 0$ in order to prove the above lemma.

Fix $(l, k) \in\left\{0,1, \ldots,\left(\begin{array}{c}n \\ k\end{array}\right)\right\} \times\{1,2, \ldots, n\}$ according to 4.2 . If $\left\{m_{1}, \ldots, m_{k}\right\}$ is the collection of specified $k$ indices for which the coordinate values of $x_{l, k}$ agree with $x$ for every $x \in L$, then denote by $L_{l, k}$ the Cartesian product $\Pi_{i=1}^{k} L_{m_{i}}$ with the usual ordering. The projection map $\Pi: L \rightarrow L_{l, k}$, defined as

$$
\Pi(x)=\left(x_{m_{1}}, \ldots, x_{m_{k}}\right),
$$

is order preserving and surjective. For each $f: L \rightarrow \mathrm{R}$, define $\tilde{f}: L_{l, k} \rightarrow \mathrm{R}$ as

$$
\tilde{f}[\Pi(x)]=f\left(x_{l, k}\right) .
$$


LemMa 4.4. Let $f: L \rightarrow \mathrm{R}$ and let $\tilde{f}$ be defined as above, then

(a) $\Delta_{m} f \geqq 0 \Rightarrow \Delta_{m} \tilde{f} \geqq 0 \quad(m=0,1, \ldots)$.

(b) If $f(x)=f\left(x_{l, k}\right)$ for every $x \in L$ then $\Delta_{m} \tilde{f} \geqq 0 \Rightarrow \Delta_{m} f \geqq 0$.

(c) $F_{l, k} \subset X_{\infty}(L)$ and $F_{l, k}$ and $X\left(L_{l, k}\right)$ are affinely isomorphic under the $\operatorname{map} f \rightarrow \tilde{f}$.

Proof. (c) The map $f \rightarrow \tilde{f}$ restricted to $F_{l, k}$ is a bijection. Let $f \in F_{l, k}$. Since $\Delta_{k} f \geqq 0$ it follows from (a) that $\Delta_{k} \tilde{f} \geqq 0$. Clearly $\tilde{f}$ satisfies 3.2 (ii) and hence by Theorem 3.6, $f$ is a CM-function on $\left(L_{l, k} ; \wedge\right)$. By (b), therefore, $f$ is a CM-function.

TheORem 4.5. The collection of completely monotonic functions on $(L ; \Lambda)$ is the cone $C_{n}(L)$. Moreover for given collections $\left\{G_{l, k}\right\}$ and $\left\{F_{l, k}\right\}$ which satisfy 4.2 , each $f \in X_{\infty}(L)$ can be written, uniquely, as a convex sum of the form

$$
f=\sum_{k=0}^{n}\left(\sum_{l=1}^{\left(\begin{array}{c}
n \\
k
\end{array}\right)} \alpha_{l, k} f_{l, k}\right)
$$

where $f_{l, k} \in F_{l, k}$. Thus the r-simplex $X_{\infty}(L)$ can be written as a direct convex sum of the closed pairwise disjoint faces $\left\{F_{l, k}\right\}$.

Proof. We only need to show that $X_{n}(L) \subset X_{\infty}(L)$. Let $f \in X_{n}(L)$. Since $G_{1,0}$ and $F_{1,0}$ are complemented in $X_{n}(L)$ by Lemma 4.3

$$
f=(1-\alpha) g+\alpha f_{1,0}
$$

where $0 \leqq \alpha \leqq 1, g \in G_{1,0}$ and $f_{1,0} \in F_{1,0}$. By the same lemma

$$
g=(1-\beta) h+\beta f_{1,1}
$$

where $h \in G_{1,1}$ and $f_{1,1} \in F_{1,1}$ and so on. The process stops after a finite number of steps since $G_{1, n}=\varnothing$ and by repeated substitution we obtain (i).

By Lemma $4.4(\mathrm{c})$, each function $f_{l, k}$ is a CM-function on $(L ; \Lambda)$ and hence $f \in X_{\infty}(L)$. Thus the $r$-simplex $X_{\infty}(L)$ can be written as a direct convex sum of the collection of closed pairwise disjoint faces $\left\{F_{l, k}\right\}$. From Alfsen [1], for given $\left\{F_{l, k}\right\}$ and $f \in X_{\infty}(L)$ it follows that the convex sum (i) is unique.

Remark. If $L=[0,1] \times[0,1]$ and $f\left(x_{1}, x_{2}\right)=\chi_{A}$ where $A=L-\{(0,0)\}$ then $\Delta_{i} f \geqq 0, i=0,1$ while

$$
\Delta_{2} f((1,1),(1,0),(0,1))=-1<0 .
$$

Math. Scand. $33-21$ 
Thus $f \notin C_{\infty}(L)$. From the example it follows that at least for $n=2$ Theorem 4.5 is "the best possible". The above theorem lead to a natural decomposition of the representing measure, $\mu_{f}$, supported by the filterspace

$$
\mathscr{F}(L)=\{F \in \mathscr{F}(L) \mid F \text { filter on }(L ; \Lambda)\}
$$

[5], for given $f \in X_{\infty}(L)$. For given $\left\{F_{l, k}\right\}$ according to 4.2 let

$$
\mathscr{F}_{l, k}(L)=\left\{F \in \mathscr{F}(L) \mid \chi_{F} \in F_{l, k}\right\}
$$

$\left(\chi_{F}\right.$ is the characteristic function of $\left.F\right)$. Then the collection $\left\{\mathscr{F}_{l, k}(L)\right\}$ consists of pairwise disjoint closed subsets of $\mathscr{F}(L)$. If $\mu_{l, k}$ is the representing measure of $f_{l, k}$ then the measures $\left\{\mu_{l, k}\right\}$ are mutually singular and hence

Corollary 4.6. Given $\left\{F_{l, k}\right\}$ according to 4.2. If $f \in X_{\infty}(L)$ is decomposed according to 4.5 (i) and if $\mu_{f}$ is $f$ 's representing measure then

$$
\mu_{f}=\sum_{k=0}^{n}\left(\sum_{l=1}^{\left(\begin{array}{l}
n \\
k
\end{array}\right)} \alpha_{l, k} \mu_{l, k}\right)
$$

where $\mu_{l, k}$ is $f_{l, k}$ 's representing measure. For given $\left\{F_{l, k}\right\}$ the measures $\left\{\mu_{l, k}\right\}$ are mutually singular and the convex sum is unique.

\section{REFERENCES}

1. E. M. Alfsen, On the geometry of Choquet simplexes, Math. Scand. 15 (1964), 97-110.

2. H. Bauer, Konvexität in topologischen Vectorräumen, Lecture notes, University of Hamburg, 1963-64.

3. N. Dunford and J. T. Schwartz, Linear Operators, Vol. I (Pure and Applied Mathematics 7), Interscience Publ., New York - London, 1958

4. N. J. Fine and P. H. Maserick, On the simplex of completely monotonic functions on a commutative semigroup, Canad. J. Math., 22 (1970), 317-326.

5. I. Kist and P. H. Maserick, BV-functions on semilattices, Pacific J. Math. 37 (1971), 711-723.

6. M. E. Munroe, Introduction to Measure and Integration, Addison Wesley Publ. co., New York, 1952.

7. R. R. Phelps, Lectures on Choquet's Theorem (Van Nostrand Mathematical Studies 7), D. Van Nostrand co., New York, 1966.

8. S. S. Wilks, Mathematical Statistics, John Wiley and Sons, Inc., 1962. 